BULL. AUSTRAL. MATH. SOC.

VOL. 24 (1981), 49-59.

\title{
MANIFOLDS OF SMOOTH MAPS II: \\ TRANSVERSALITY
}

\section{TRUONg CôNg NGHE}

\begin{abstract}
We generalise the standard notion of transversality to maps between manifolds modelled on locally convex spaces and prove that all standard transversal theorems remain valid for this generalised notion.

As first applications, we use our generalised Transversal Density Theorem to prove several simple "generic" results for local smooth maps which parallel the usual ones.
\end{abstract}

This is a sequel of [5]. In this paper, we restrict out interest to the $B \Gamma$-manifolds [5] and define a notion of transversality for maps between them. We term it the $B \Gamma$-transversality which is in fact a generalisation of the standard notion [1], [2], [3]. We then show that all the standard transversal theorems [1], [2], [3] still hold for this generalised notion. Finally, as first applications, we give several simple "generic" results for local smooth maps which parallel the usual ones [2], [3], [4].

The paper consists of three sections. In $\$ 1$, we define the $B \Gamma-$ transversality and give its standard properties. The next section, $\$ 2$, is for stating and proving the $B \Gamma$-transversal theorems and the last section is for the applications.

Received 12 February 1981. 


\section{1. $B \Gamma$-transversality}

In the following, we shall always assume that $F$ is a $\Gamma$-family consisting of sequentially complete spaces. First, in exactly the same way as in the case of Banach manifolds, we can define $B \Gamma$-submanifolds and $B \Gamma$-products of $B \Gamma$-manifolds of class $C^{r}$ modelled on $F$. Now, let $X, Y$ be $B \Gamma$-manifolds of class $C^{r}$ modelled on $E, F \in F$ respectively $(r \geq 1)$, and $W$ be a $B \Gamma$-submanifold of class $C^{r}$ of $Y$. Then a $C_{B \Gamma^{-}}^{r}$ map [5] $f: X \rightarrow Y$ is said to be $B \Gamma$-transversal to $W$ at $x \in X$ if either $f(x) \vDash W$ or $y=f(x) \in W$ and satisfies the following condition: there exists a $B \Gamma$-complement $Z$ of $T_{y} W$ in $T_{y} Y$ such that, if we denote by $Q: T_{y} W \oplus Z \rightarrow Z$ the projection, the map $Q \circ T_{x} f: T_{x} X+Z$ is a $B \Gamma$-splitting surjection [6], [7]. We say that $f$ is $B \Gamma$-transversal to $W$ if it is $B \Gamma$-transversal to $W$ at every $x \in X$.

(1.1) (Local Representative of $B \Gamma$-transversality [1]). Let $X, Y, W$ and $f: X \rightarrow Y$ be as above and assume that $x \in X$ is such that $y=f(x) \in W$. Then a necessary and sufficient condition for $f$ to be $B \Gamma$-transversal to $W$ at $x$ is: there exist admissible Br-charts $(U, \alpha)$ and $(V, B)$ at $x$ and $f(x)$ respectively, $B \Gamma$-decompositions $E=E_{1} \oplus_{B \Gamma} E_{2}, \quad F=F_{1} \oplus_{B \Gamma} F_{2}$ for $E$ and $F$ such that:

(a) $\alpha(U)=B_{1}+B_{2} \subseteq E, \quad \beta(V)=D_{1}+D_{2} \subseteq F, \alpha(x)=0$, $B(y)=0, \quad \beta(W \cap V)=D_{1}$, where $B_{i}$ and $D_{i}$ are open neighbouthoods of 0 in $E_{i}, F_{i}(i=1,2)$ respectively;

(b) the Zocal representative $f_{\alpha \beta}: B_{1}+B_{2} \rightarrow D_{1}+D_{2}$ of $f$ has the form

$$
f_{\alpha \beta}=\eta+\Phi \circ P_{2}
$$

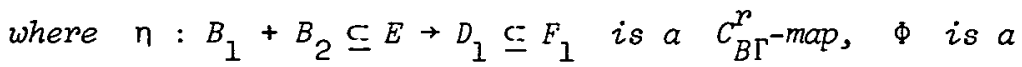
$B \Gamma$-isomorphism of $E_{2}$ onto $F_{2}$ and $P_{2}: E=E_{1} \oplus_{B \Gamma} E_{2} \rightarrow E_{2}$ is the second projection.

Proof. Straightforward with the use of the Inverse Mapping Theorem $[7,(5.2)$, p. 45]. 
(1.2). Let $X, Y, W$ and $f: X \rightarrow Y$ be as above and suppose that $f$ is $B \Gamma$-transversal to $W$. Then $f^{-1}(W)$ is either empty or a $B \Gamma$-submanifold of class $C^{r}$ of $X$. In the latter case, we have

(a) for $x \in f^{-1}(W)$ and $y=f(x), \quad\left(T_{x} f\right)^{-1}\left(T_{y} W\right)=T_{x}\left(f^{-1}(W)\right)$,

(b) $W$ and $f^{-1}(W)$ have the same codimension.

Proof. This follows from (1.1).

\section{The $B \Gamma$-transversal theorems}

Let $F$ be a $\Gamma$-family which contains $E, F, G$, and $F \times G$ which is a $B \Gamma$-product with the projection $\pi: F \times G+F$ and $E \subseteq F \times G$. Then $E$ is said to $B \Gamma$-adapt the projection $\pi$ if there is a $B \Gamma$-complement $H$ of $E$ in $E \times G$ such that $F=\pi(E) \oplus \pi(H)$ is a $B \Gamma$-direct sum when both summands are equipped with the relative calibrations from $\Gamma_{F}$.

(2.i). Let $E, F, G$ be as above and assume that $E$ BI-adapts $\pi$. If $\operatorname{dim} G=n$ and $\operatorname{codim} E=q$ (with respect to $F \times G$ ) then the restriction of $\pi$ to $E$ is a $B \Gamma-F r e d h o$ m map with index $n-q$ [6].

Proof. Let $H$ be the $B \Gamma$-complement of $E$ in the definition of $B \Gamma$-adaptation and $P, Q$ be the corresponding projections of the $B \Gamma$-decomposition $F \times G=E \oplus_{B \Gamma} H$.

Put $L=P \pi(E)$ and $K=E \cap(\{0\} \times G)$. Then it is easily seen that $E=K \oplus_{B \Gamma} L$ and $L$ is $B \Gamma$-isomorphic to $\pi(E)$ and the proof follows.

Now let $A, X, Y$ be $B \Gamma$-manifolds modelled respectively on $E, F, G \in F$. Suppose that $E \times F$ is a $B \Gamma$-product and denote by $A \times X$ the $B \Gamma$-product of $A$ and $X$ (which is modelled on $E \times F$ ). Let $c_{B \Gamma}^{\dot{r}}(X, Y)$ be the space of $c_{B \Gamma}^{r}$-maps $X \rightarrow Y$. Then, a map $\rho: A \rightarrow C_{B \Gamma}^{p}(X, Y)$ is called a $C_{B \Gamma}^{p}$-representation [1] if and only if the evaluation map $\mathrm{ev}_{\rho}: A \times X \rightarrow Y$ defined by $\operatorname{ev}_{\rho}(a, x)=\rho(a)(x)$ for $a \in \mathrm{A}, x \in X$ is a $C_{B \Gamma^{-m a p}}^{r}$

(2.2) (BГ-Transversal Density Theorem [1]). Let $F$ be a $\Gamma$-family 
as at the beginning. Let $E, F, E \times F \in F$ where $E \times F$ is a $B \Gamma$-product. Let $A$ be a second countable $B \Gamma$-manifold modelled on $E, X$ be a second countable BГ-manifold modelled on $F$ with finite dimension $n$ and $Y$ be a BI-manifold modelzed on $G$, alz of class $C^{r}$. Let $\rho: A \rightarrow c_{B \Gamma}^{r}(X, Y)$ be a $c_{B \Gamma}^{r}$-representation and $W$ be a $B \Gamma$-submanifold of class $c^{r}$ of $y$ with finite codimension $q$ such that $r>\max (0, n-q)$.

Now assume that $\mathrm{ev}_{\rho}$ is $B \Gamma$-transversal to $W$ and, for any $(a, x) \in A \times X$ such that $y=\rho(a)(x) \in W$, the subspace $\left(T_{(a, x)} \mathrm{ev}_{\rho}\right)^{-1}\left(T_{y} W\right) \quad B \Gamma$-adapts the projection $T_{a} \mathrm{~A} \times T_{x} X T_{a} \mathrm{~A}$. Then the set of $a \in A$ such that $\rho(a)$ is $B \Gamma$-transversal to $W$ is residual in A.

Proof. The proof is similar to the proof in [1, Theorem 19.1] with the use of (2.1).

(2.3) (Openness of $B \Gamma$-transversality [1]). Let $F$ be a $\Gamma$-famizy, $E, F, G \in F$ such that $E \times F$ and $F \times G$ are $B \Gamma$-products. Let $A, X, Y$ be $B \Gamma$-manifolds of class $C^{l}$ modelzed on $E, F, G$ respectively. Let $W$ be a closed $B \Gamma$-submanifold of $Y$ of class $C^{l}, K \subseteq X$ a compact subset and $\rho: A \rightarrow C_{B \Gamma}^{1}(X, Y)$ a $C_{B \Gamma}^{1}$-representation. Then the set of $a \in A$ such that $\rho(a)$ is $B \Gamma$-transversal to $W$ at every $x \in K$ is open in $A$.

Proof. First, in the same manner as in the case of Banach manifolds, we can define the $B \Gamma$-bundle $L_{B \Gamma}\left(\tau_{X}, \tau_{Y}\right)$ of $B \Gamma$-continuous linear maps whose fibre over a point $(x, y) \in X \times Y$ (the $B \Gamma$-product of $X$ and $Y$ ) is the space $L_{B \Gamma}\left(T_{x}^{X}, T_{y} Y\right)$ of $B \Gamma$-continuous linear maps from $T_{x} X$ to $T_{y} Y$. Then we proceed exactly as in [1].

Now let $F$ be a $\Gamma$-family containing $E, F, G$ and assume that $E \times F$ and $E \times G$ are $B \Gamma$-products. Let $A, X, Y$ be $B \Gamma$-manifolds of class $c^{r+1}(r \geq 1)$ modelled on $E, F, G$ respectively.

Suppose that $X$ is compact (hence $F$ is finite-dimensional), and let 
$\rho: A \rightarrow C_{B \Gamma}^{r+1}(X, Y)$ be a $C_{B \Gamma}^{r+1}$-representation, $W \subseteq Y$ be a closed $B \Gamma$-submanifold of class $c^{r+1}$, and $a_{0} \in A$ be a point such that $\rho\left(a_{0}\right)$ is $B \Gamma$-transversal to $W$. For each $a \in A$, let $w_{a}=\rho(a)^{-1}(W) \subseteq X$. Then, by (2.3), for all $a \in A$ sufficiently near $a_{0}, \rho(a)$ is $B \Gamma$-transversal to $W$. Hence, by $(1.2), W_{a}$ is a $B \Gamma$-submanifold of class $c^{r+1}$ of $X$ (that is, a $c^{r+1}$-submanifold of $X$ in the Banach sense since $X$ is a finite-dimensional manifold). Thus one might expect that, for $a$ near $a_{0}$, the submanifolds $W_{a}$ and $W_{a_{0}}$ are close. Indeed, we have

(2.4) (BГ-Transversal Isotopy Theorem [1]). Let $F$ be a $\Gamma$-family containing $E, F, G$ such that $E \times F$ and $F \times G$ are $B \Gamma$-products. Let $A, X, Y$ be $B \Gamma$-manifolds of class $C^{r+1}(r \geq 1)$ modelied on $E, F, G$ with $X$ compact and of class $C^{p+3}$. Let $\rho: A \rightarrow T_{B \Gamma}^{p+1}(X, Y)$ be a $c_{B \Gamma}^{r+1}$-representation and $W \subseteq Y$ be a closed $B \Gamma$-submanifold of class $c^{x+1}$. Finalzy, let $a_{0} \in A$ be a point such that $\rho\left(a_{0}\right)$ is $B \Gamma$-transversal to $W$. Then there is an open neighbourhood $N$ of $a_{0}$ in A such that, for all $a \in N$, the submanifold $W_{a}=\rho(a)^{-1}(W)$ is $c^{r}$-isotopic to $w_{\alpha_{0}}$.

Proof. Since $X$ is compact, hence of finite dimension, the proof of [1, Theorem 20.2] can be repeated with suitable modifications.

\section{Applications}

In this section, as first applications of the $B \Gamma$-Transversal Density Theorem (2.2), we give some simple "generic" results for local smooth maps which parallel the usual ones [2], [3], [4].

From now on, $\Omega$ shall always stand for an open, convex and bounded subset of an Euclidean space $\mathbb{R}^{n}$, and for each integer $k \geq 0, P^{k}(n, m)$ shall stand for the space of polynomials of degree less than or equal to $k$ 
from $\mathbf{R}^{n}$ to another Euclidean space $\mathbf{R}^{m}$ [1, p. 23]. For each $\xi \in C^{k}\left(\Omega, \mathbf{R}^{m}\right)$ and each $x \in \Omega, P^{k} \xi(x)$ is the point of $P^{k}(n, m)$ given by [1]:

$$
p^{k} \xi(x)=\left(\xi(x), D \xi(x), \ldots, D^{k} \xi(x)\right) .
$$

We choose the following norm on $P^{k}(n, m)$. If $\left(a_{0}, a_{1}, \ldots, a_{k}\right) \in P^{k}(n, m)$ then

$$
\left\|\left(a_{0}, a_{1}, \ldots, a_{k}\right)\right\|=\left\|a_{0}\right\|+\left\|a_{1}\right\|+\ldots+\left\|a_{k}\right\|,
$$

and, for each $\xi \in C^{k}\left(\Omega, \mathbf{R}^{m}\right)$, define

$$
\|\xi\|_{k}=\sup \left\{\left\|P^{k} \xi(x)\right\|: x \in \Omega\right\} \text {. }
$$

For each integer $k \geq 0$, let $B^{k}\left(\Omega, \mathbf{R}^{m}\right)$ denote the space of all $\xi \in C^{k}\left(\Omega, \mathbb{R}^{m}\right)$ such that $\|\xi\|_{k}<+\infty$, and put

$$
B^{\infty}\left(\Omega, \mathbf{R}^{m}\right)=\bigcap_{k=0}^{\infty} B^{k}\left(\Omega, \mathbf{R}^{m}\right)
$$

Endow $B^{\infty}\left(\Omega, \mathbf{R}^{m}\right)$ with the calibration consisting of the following sequence of increasing norms:

$$
\Gamma=\left\{\|\cdot\|_{k}: k \geq 0\right\}
$$

where each $\|\cdot\|_{k}$ is defined by (3).

(3.1). The space $B^{\infty}\left(\Omega, \mathbf{R}^{m}\right)$ equipped with the sequence of increasing norms in (5) is a separable Fréchet space.

Proof. We need only prove separability. For each $k \geq 0$, define the space

$U b^{k}\left(\Omega, \mathbf{R}^{m}\right)=\left\{f: \Omega \rightarrow \mathbf{R}^{m} / f \in B^{k}\left(\Omega, \mathbf{R}^{m}\right)\right.$ and $f, D f, \ldots, D^{k} f$ are uniformly continuous on $\Omega\}$.

Then, for every $k \geq 0, U B^{k}\left(\Omega, \mathbf{R}^{m}\right)$ is a Banach space. Furthermore, from the fact that $U B^{0}\left(\Omega, \mathbf{R}^{m}\right)$ is separable, using the map 


$$
P^{k}: U B^{k}\left(\Omega, \mathbb{R}^{m}\right)+U B^{0}\left(\Omega, P^{k}(n, m)\right): f \rightarrow P^{k} f,
$$

it is seen that $U B^{k}\left(\Omega, \mathbb{R}^{m}\right)$ is separable for all $k \geq 0$. From this, $B^{\infty}\left(\Omega, \mathbb{R}^{m}\right)$ is separable.

Now note that each $\|\cdot\|_{j}$ on $B^{\infty}\left(\Omega, \mathbb{R}^{m}\right)$ induces a norm $p_{j}$ on $B^{\infty}\left(\Omega, \mathbf{R}^{m}\right) \times \mathbb{R}^{n}$ defined by

$$
p_{j}(\xi, x)=\|\xi\|_{j}+\|x\| \text { for }(\xi, x) \in B^{\infty}\left(\Omega, \mathbb{R}^{m}\right) \times \mathbf{R}^{n} .
$$

(3.2). Let $\Omega$ be as above. Let $r$ be an integer greater than or equal to 1 and $k$ be an integer such that $0 \leq k \leq r$. Then we can always find an integer $i_{0}$ such that the following assertions are true $[6],[7]:$

(a) the map ev $\operatorname{ev}_{k}: B^{\infty}\left(\Omega, \mathbf{R}^{m}\right) \times \Omega \rightarrow \Omega \times P^{k}(n, m)$ defined by $\mathrm{ev}_{k}(\xi, x)=\left(x, p^{k} \xi(x)\right)$ for $x \in \Omega, \xi \in B^{\infty}\left(\Omega, \mathbb{R}^{m}\right)$ is $C_{B \Gamma}^{p}$ with respect to the calibration

$$
\Gamma=\left\{\left(p_{r+k+i},\|\cdot\|\right): i \geq i_{0}\right\}
$$

for the pair $\left(B^{\infty}\left(\Omega, \mathbb{R}^{m}\right) \times \mathbb{R}^{n}, \mathbb{R}^{n} \times P^{k}(n, m)\right)$, where $\|\cdot\|$ is the standard norm of $\mathbf{R}^{n} \times P^{k}(n, m)$;

(b) for each $(\xi, x) \in B^{\infty}\left(\Omega, \mathbf{R}^{m}\right) \times \Omega$, the $B \Gamma$-derivative $\operatorname{Dev}_{k}(\xi, x): B^{\infty}\left(\Omega, \mathbf{R}^{m}\right) \times \mathbf{R}^{n} \rightarrow \mathbf{R}^{n} \times p^{k}(n, m)$ is onto and has its kermel $B \Gamma$-splitting in $B^{\infty}\left(\Omega, \mathbb{R}^{m}\right) \times \mathbb{R}^{n}$;

(c) for any (BГ-splitting) subspace $F_{1}$ of $\mathbb{R}^{n} \times P^{k}(n, m)$, the inverse image $E_{1}=\operatorname{Dev}_{k}(\xi, x)^{-1}\left(F_{1}\right)$ is ${ }_{B} \Gamma_{\text {-splitting }}$ in $B^{\infty}\left(\Omega, \mathbf{R}^{m}\right) \times \mathbf{R}^{n}$ and has a $B \Gamma$-complement $E_{2}$ such that the restriction of $\operatorname{Dev}_{k}(\xi, x)$ to $E_{2}$ is a $B \Gamma$-isomorphism 
onto a BГ-complement $F_{2}$ of $F_{1}$ in $\mathbb{R}^{n} \times P^{k}(n, m)$;

(d) the subspace $E_{1}=\operatorname{Dev}_{k}(\xi, x)^{-1}\left(F_{1}\right)$ BI-adapts the first projection $B^{\infty}\left(\Omega, \mathbf{R}^{m}\right) \times \mathbf{R}^{n} \rightarrow B^{\infty}\left(\Omega, \mathbf{R}^{m}\right)$.

Proof. (a) follows from the remark in $[7$, p. 26].

(b) The surjectivity of $\operatorname{Dev}_{k}(\xi, x)$ is seen as in the proof of [1, Theorem 10.4, p. 27]. Now, for a fixed $(\xi, x) \in B^{\infty}\left(\Omega, \mathbf{R}^{m}\right) \times \Omega$, defined [1],

$$
\begin{aligned}
& K_{1}=\left\{\zeta \in B^{\infty}\left(\Omega, \mathbf{R}^{m}\right) / D^{i} \zeta(x)=0 \text { for } i=0,1, \ldots, k\right\}, \\
& K_{2}=\left\{\zeta \in B^{\infty}\left(\Omega, \mathbf{R}^{m}\right) / D^{i} \zeta \equiv 0 \text { for } i \geq k+1\right\} .
\end{aligned}
$$

Then it is not hard to see that $K_{2}=P^{k}(n, m), B^{\infty}\left(\Omega, \mathbf{R}^{m}\right)=K_{1} \oplus_{B \Gamma} K_{2}$ and $B^{\infty}\left(\Omega, \mathbb{R}^{m}\right) \times \mathbb{R}^{n}=\left(K_{1} \times\{0\}\right) \oplus_{B \Gamma}\left(K_{2} \times \mathbb{R}^{n}\right)$. From this we have $(b)$.

(c) Let $F_{1}$ be a subspace of $\mathbf{R}^{n} \times P^{k}(n, m)$; then we have $K_{1} \times\{0\}=\operatorname{Ker} \operatorname{Dev}_{k}(\xi, x) \subseteq E_{1}=\operatorname{Dev}_{k}(\xi, x)^{-1}\left(F_{1}\right)$. From this it follows quickly that, if $L=E_{1} \cap\left(K_{2} \times \mathbb{R}^{n}\right)$, we have

$$
E_{1}=L \Theta_{B \Gamma}\left(K_{1} \times\{0\}\right) \text {. }
$$

Since $\operatorname{codim} L<+\infty$ and $K_{2} \times R^{n}$ is a normed space with norm $\|(\zeta, h)\|=\|\zeta\|_{k}+\|h\|$, we can find a complement $E_{2}$ of $L$ in $K_{2} \times \mathbf{R}^{n}$ such that

$$
\pi(L) \oplus_{B \Gamma} \pi\left(E_{2}\right)=K_{2} \quad(\text { see }[1])
$$

where $\pi: B^{\infty}\left(\Omega, \mathbf{R}^{m}\right) \times \mathbf{R}^{n} \rightarrow B^{\infty}\left(\Omega, \mathbf{R}^{m}\right)$ is the first projection.

Then, by a simple argument, we can prove $(c)$.

(d) It is not hard to verify that

$$
\pi\left(E_{1}\right) \Theta_{B \Gamma} \pi\left(E_{2}\right)=B^{\infty}\left(\Omega, \mathbf{R}^{m}\right)
$$


which proves that $E_{1} B \Gamma$-adapts $\pi$.

Let $\Omega$ be as above and let $B^{\infty}(\Omega, R)$ be the space of all $C^{\infty}$ functions $\Omega \rightarrow \mathbf{R}$ with all derivatives bounded on $\Omega$. Let us denote by $M^{\infty}(\Omega, \mathbf{R})$ the subset of Morse functions [2], [3] in $B^{\infty}(\Omega, \mathbb{R})$.

(3.3). $M^{\infty}(\Omega, \mathrm{R})$ is dense in $B^{\infty}(\Omega, \mathrm{R})$. In other words, every function in $B^{\infty}(\Omega, R)$ can be approximated by Morse functions.

Proof. Let $J^{\mathcal{I}}(\Omega, \mathbf{R})$ be the space of 1 -jets $\Omega+\mathbf{R}$ and let

$$
S_{1}=\left\{\sigma \in J^{1}(\Omega, \mathbb{R}): \operatorname{corank} \sigma=1\right\}
$$

be the submanifold of $J^{l}(\Omega, R)$ defined in $[2$, p. 60]; then

$$
M^{\infty}(\Omega, \mathbf{R})=\left\{f \in B^{\infty}(\Omega, \mathbf{R}): j^{l} f \ldots S_{1}\right\} \text {. }
$$

From this we can apply (2.2) with $A=B^{\infty}(\Omega, \mathbf{R})$ considered as a $B \Gamma$-manifold, $X=\Omega \subseteq \mathbb{R}^{n}, \quad Y=J^{1}(\Omega, \mathbb{R}), W=S_{1}$ and $\rho: A \rightarrow C_{B \Gamma}^{1}\left(\Omega, J^{1}(\Omega, R)\right)$ defined by $\rho(f)=j^{1} f$, where $f \in A$ and $j^{I} f: \Omega \rightarrow J^{I}(\Omega, \mathbb{R})$ is the l-jet prolongation of $f[3]$.

Now consider the tangent bundle $R=\Omega \times \mathbb{R}^{n}$ and the space of all $C^{\infty}$ sections of $T \Omega$. Each element of the latter space is a vector field $\xi: \Omega+T \Omega$ defined by

$$
\xi(x)=(x, \tilde{\xi}(x)) \text { for all } x \in \Omega
$$

where $\tilde{\xi}: \Omega \rightarrow \mathbf{R}^{n}$ is $C^{\infty}$.

Denote by $S^{\infty}(T \Omega)$ the space of all such $\xi$ with $\tilde{\xi} \in B^{\infty}\left(\Omega, \mathbf{R}^{n}\right)$. Consider the usual topology on $B^{\infty}\left(\Omega, \mathbb{R}^{n}\right)$ defined by (5), and for each $k \geq 0$, defined

$$
\|\xi\|_{k}=\|\tilde{\xi}\|_{k} \text { for all } \xi \in S^{\infty}(T \Omega) \text {. }
$$

Then $S^{\infty}(T \Omega)$ equipped with $\left\{\|\cdot\|_{k}: k \geq 0\right\}$ is a separable Fréchet space. We say that $\xi \in S^{\infty}(T \Omega)$ is a o-transversal vector field on $\Omega$ $[1$, p. 62] if and only if every critical point of $\xi$ is non-degenerate. 
(3.4). The set $G_{0} \subseteq S^{\infty}(T \Omega)$ of 0 -transversal vector fiezds on $\Omega$ is dense in $S^{\infty}(T \Omega)$.

Proof. Similar to the one of (3.3).

REMARK. It is clear that each $\xi \in G_{0}$ has isolated critical points. Thus (3.4) also shows that there is a dense subset $G_{0} \subseteq S^{\infty}(T \Omega)$ such that every $\xi \in G_{0}$ has only isolated critical points. Indeed, we can prove it directly as follows.

(3.5). There is a dense subset $G \subseteq S^{\infty}(T \Omega)$ such that every $\xi \in G$ has only isolated zeros.

Proof. Apply the Infinite Codimension Lemma [4] to find an integer $k$ so large that

$$
q=\operatorname{codim}\left(W^{k} \text { in } J^{k}(n, n)\right)>n
$$

where $W^{k}$ is the algebraic subset constructed in [4, p. 140].

Define $\tilde{W}=\Omega \times W^{k} \subseteq \Omega \times J^{k}(n, n)=J^{k}(T \Omega)$ and write $\tilde{W}$ as a finite union of submanifolds of $J^{k}(T \Omega)$ of codimension greater than $n$. Then apply (2.2) to each member of the union.

Let $\Omega \subseteq \mathbb{R}^{n}$ be as before and let $f \in B^{\infty}\left(\Omega, \mathbf{R}^{n}\right)$. We say that $f$ is locally finite-to-one [4] if and only if every point $x \in \Omega$ has a neighbourhood $U \subseteq \Omega$ such that $f^{-1}(y) \cap U$ is finite for all $y \in \mathbf{R}^{n}$.

(3.6). There is a dense subset $G \subseteq B^{\infty}\left(\Omega, \mathbf{R}^{n}\right)$ such that each $f \in G$ has the property that $f: \Omega \rightarrow \mathbf{R}^{n}$ is zocally finite-to-one.

Proof. Similar to the one of (3.5).

Our last application is concerned with fixed points of $c^{\infty}$ maps $f \in B^{\infty}\left(\Omega, \mathbf{R}^{n}\right)$

(3.7). There is a dense subset $G \subseteq B^{\infty}\left(\Omega, \mathbf{R}^{n}\right)$ such that every $f \in G$ has only isolated fixed points.

Proof. As the one of (3.6). 


\section{References}

[1] Ralph Abraham, Joel Robbin, Transversal mappings and flows (Benjamin, New York, Amsterdam, 1967).

[2] M. Golubitsky, V. Guillemin, Stable mappings and their singularities (Graduate Texts in Mathematics, 14. Springer-Verlag, New York, Heidelberg, Berlin, 1973).

[3] Morris W. Hirsch, Differential topology (Graduate Texts in Mathematics, 33. Springer-Verlag, New York, Heidelberg, Berlin, 1976).

[4] H. Kurland and J. Robbin, "Infinite codimension and transversality", Dynamical Systems, 135-150 (Proc. Sympos. University of Warwick, 1973/74. Lecture Notes in Mathematics, 468. Springer-Verlag, Berlin, Heidelberg, New York, 1975).

[5] Truong Công Nghê, "Manifolds of smooth maps", BuzZ. Austraz. Math. Soc. 24 (1981), 1-11.

[6] Truong Công Nghê and S. Yamamuro, "Locally convex spaces, differentiation and manifolds", Comment. Math. Special Issue 2 (1979), 229-338.

[7] Sadayuki Yamamuro, A theory of differentiation in locally convex spaces (Memoirs of the American Mathematical Society, 212. American Mathematical Society, Providence, Rhode Island, 1979).

Department of Pure Mathematics, University of Sydney, Sydney, New South Wales 2006, Australia. 